Review

\title{
Hair Structures Affecting Hair Appearance
}

\author{
Shinobu Nagase
}

Hair Care Products Research, Kao Corporation, Tokyo 131-8501, Japan; nagase.shinobu@kao.com

Received: 15 June 2019; Accepted: 9 July 2019; Published: 11 July 2019

check for updates

\begin{abstract}
Optical factors affecting hair appearance are reviewed based on hair structures from macroscopic to microscopic viewpoints. Hair appearance is the result of optical events, such as reflection, refraction, scattering, and absorption. The effects of hair structures on such optical events are summarized and structural conditions for hair appearance are considered. Hair structures are classified into the following: the alignment of multiple hair fibers, the cross-sectional shape of the hair fiber, and the microstructures of hair fiber (cuticle, cortex, and medulla). The alignment of multiple hair fibers is easily affected by the existence of meandering fibers and their alignment along hair length becomes less-synchronized. The less-synchronized orientation of multiple fibers causes the broadening of the apparent reflection and luster-less dull impression. The cross-sectional shape of hair fiber affects light reflection behavior. Hair fibers with elliptical cross-section show glittering colored light based on total reflection in the hair. The scaly structures of cuticles at the surface of hair are often uplifted and cause light scattering, and then affect hair luster. The porous structure of the cortex and medulla in hair fiber can cause light scattering and affect hair luster and color. The above phenomena suggest that important factors for hair appearance are the alignment of multiple hair fibers, appropriate cross-sectional shape, ordered scaly structure, and pore-less internal structure.
\end{abstract}

Keywords: hair; appearance; structure; alignment; cross-section; cuticle; cortex; medulla

\section{Introduction}

Hair appearance is an important factor for the fascinating impression of human beings. Hair appearance is varied depending on hair types, such as color and shape, and on hair treatments like coloring and perming, and also on undesirable damage by various stimuli. These variations in hair appearance are caused by optical properties like reflection, refraction, scattering, and absorption. "Reflection" and "refraction" are light reflection and refraction at the interface between two components with different refractive indices, respectively, and "scattering" and "absorption" are light scattering and absorption, respectively, by some structures on and in the hair fiber, like micro-pores and melanin granules. It is important, therefore, to investigate and understand the relationship between those optical properties and the structure of hair fiber based on a wide viewpoint from macroscopic to microscopic levels.

The effect of hair structures on hair appearance, especially on hair luster, has been studied by many scientists [1-22]. Initial approaches have been focused on the effect of the cuticle structure in many cases, because the cuticle is located on the outermost surface of hair fiber and has been reasonably considered as the most important factor for the reflection [5-8]. Some oils and/or polymers have also been used to enhance light reflection at the hair surface and to improve hair luster $[7,8]$. On the other hand, the effect of hair internal structure on hair appearance has been studied by various research groups [9-16]. This effect of internal structure is considered as smaller than the effect of surface cuticles, in the case of dark colored hair, while the effect of internal structure is more significant and not negligible, in the case of lighter colored hair. 
The quantitative evaluation of hair appearance (luster) has been studied and various methods for the evaluation have been proposed based on goniophotometric measurements $[2,3,5-7,9,10,12,13,17]$ and on digital imaging methods $[4,8,18,20,22]$ including computer graphics [19,23]. The digital imaging methods are useful to understand the relationship between hair structure and hair appearance intuitively via two-dimensional image analyses. In this article, the effects of various hair structures on hair appearance are qualitatively discussed based on typical digital images showing the effects of the structures.

In this article, I summarize the effects of several hair structures on optical properties and on hair appearance. In the following sections, the effects of the alignment of multiple hair fibers, of the cross-sectional shape of the hair fiber, and of the surface and internal structures of the fiber are summarized based on the previously established works by our research group. Structural conditions for hair appearance are then discussed and considered. I hope these summary and considerations are helpful to understand the mechanisms of hair appearance based on hair structure, and to develop significant new technologies to improve hair appearance.

Knowledge of the optical properties of hair fiber is important, not only for cosmetic science, but also for computer graphics to reconstruct realistic hair images [19].

\section{Alignment of Multiple Hair Fibers}

Hair alignment is one of the important properties for hair appearance. There are articles that report the effect of hair alignment on hair appearance [20-22,24]. In articles [21,22], the effect of aging on hair appearance was observed in 230 Japanese female panelists aged from 10 to 70 . The result of hair luster evaluation is shown in Figure 1. There is a clear tendency for younger panelists to be evaluated as "lustrous" to "somewhat lustrous", while there was a tendency for older panelists to be evaluated as "luster-less" to "somewhat luster-less", especially panelists more than 45 years old. In this case, all panelists had not experienced perming to change their hair style.

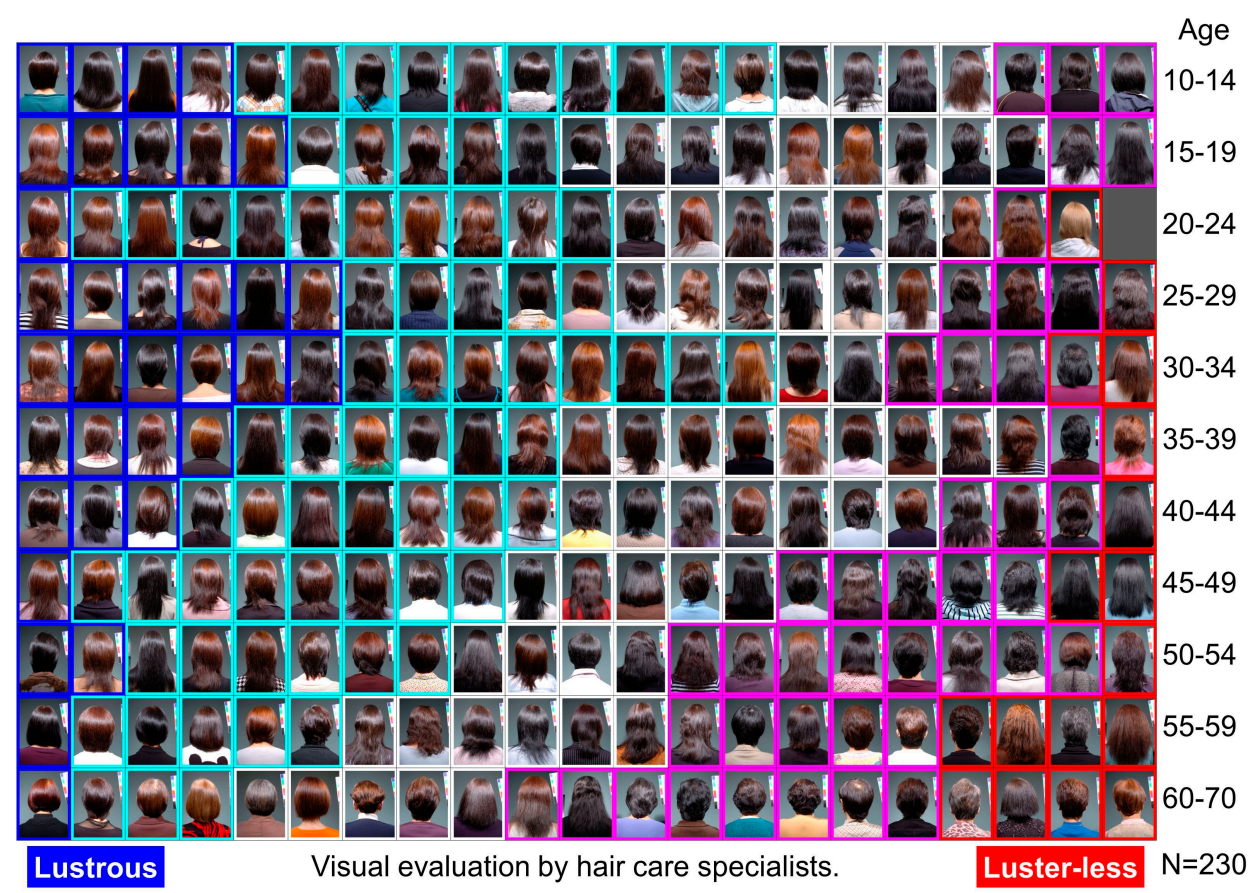

Figure 1. Variation and distribution of hair appearance (hair luster) in 230 Japanese panelists (female, aged 10-70). Hair luster was visually evaluated in five steps from luster-less to lustrous, by three hair care specialists (one hair stylist and two hair scientists), under constant lighting conditions just after shampooing followed by hair drying. "Luster-less" is shown in red, "somewhat luster-less" is pink, "in-between" is white, "somewhat lustrous" is sky blue, and "lustrous" is blue, in this picture. 
Since the photographs in Figure 1 are too small to see their appearance, the larger photographs of typical examples are shown in Figure 2, according to hair length and color in each generation. The difference in hair appearance between neighboring generations is not so significant; however, gradual change in the hair appearance is observable from age 10-60 in Figure 2. Younger panelists show relatively sharp and clear hair luster, while older panelists tend to show diffused unclear hair luster. Those differences in hair luster were analyzed more in detail.

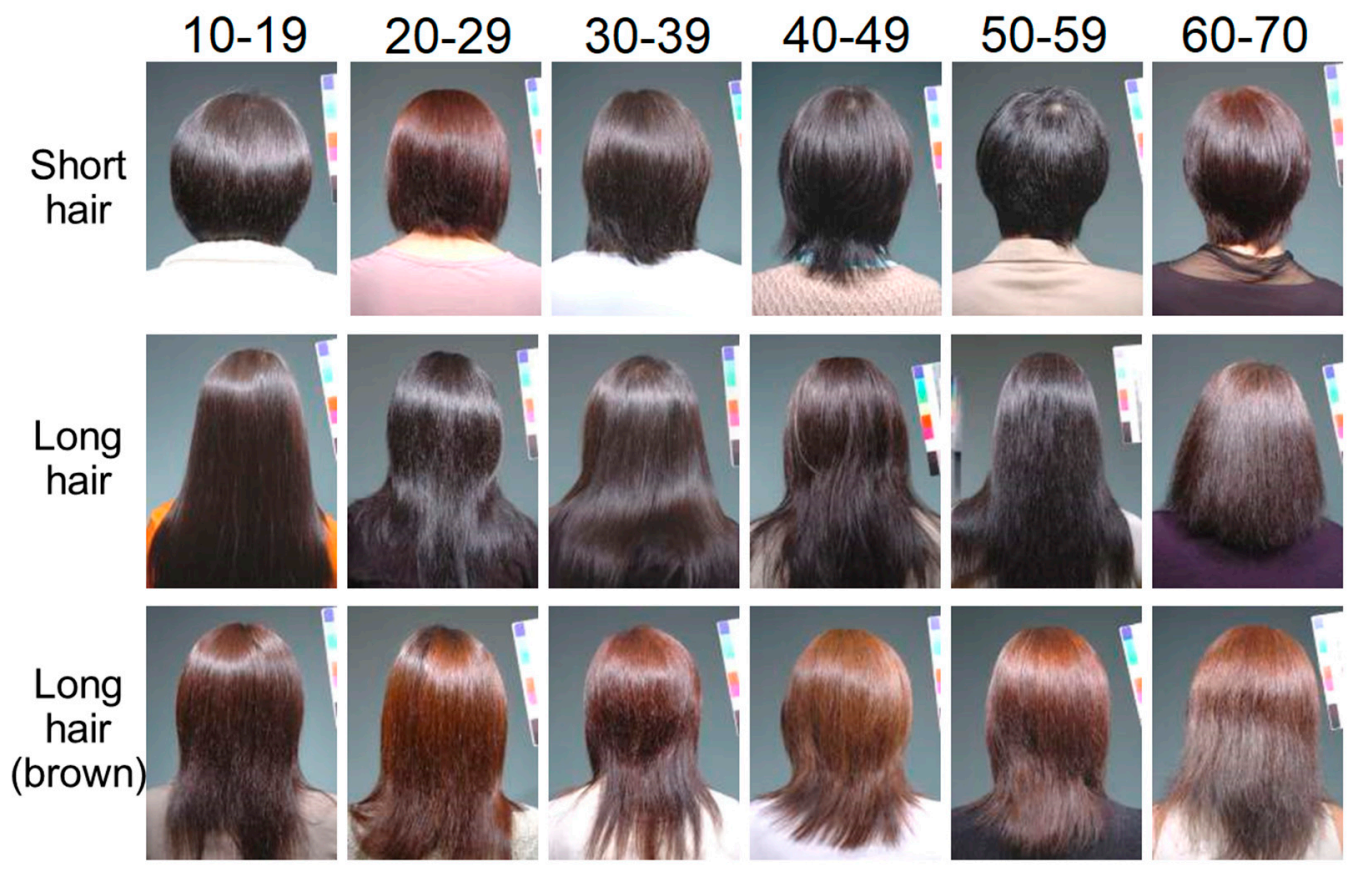

Figure 2. Typical examples for change in hair appearances from Japanese female panelists aged 10-60. Those examples are roughly classified according to hair length and color. It seems that hair appearance is possibly affected by age as well as hair color and length.

In order to investigate the reason for the change in hair luster accompanied with age, the hair was observed in detail. The enlarged photographs of three typical examples of panelists of 25, 40, and 57 years old are shown in Figure 3. The photograph of hair fibers sampled from each panelist is also shown in the figure. Panelist A (aged 25) has more straight hair fibers and shows aligned multiple hair fibers, which have almost synchronized orientation along hair length, and, therefore, relatively sharp luster can be observed. Hair fibers sampled from older panelists become more meandering, and, therefore, the alignment of multiple hair fibers becomes less-synchronized along the hair length. This is probably the reason why hair appearance becomes luster-less.

Figure 3 clearly shows the effect of hair alignment on hair appearance; however, there are other possibilities for the reason of the change in hair appearance with age, such as hair damage by coloring for gray hair and/or the effect of hair length as seen in Figure 2. The hairs of panelists shown in Figure 3 were actually colored with oxidative colorants within three months before the observation. Therefore, the effects of the meandering hair fibers on hair alignment and on appearance were verified by model hair bundles made of straight and meandering fibers.

Figure 4 shows the appearances of hair bundles of only straight hair, of $5 \%$ meandering hair in the straight hair, and of $10 \%$ meandering hair [24]. Hair alignment and appearance are clearly changed by adding meandering fibers. The above results of hair observations show that the alignment of multiple hair fibers is an important factor to control hair appearance. 


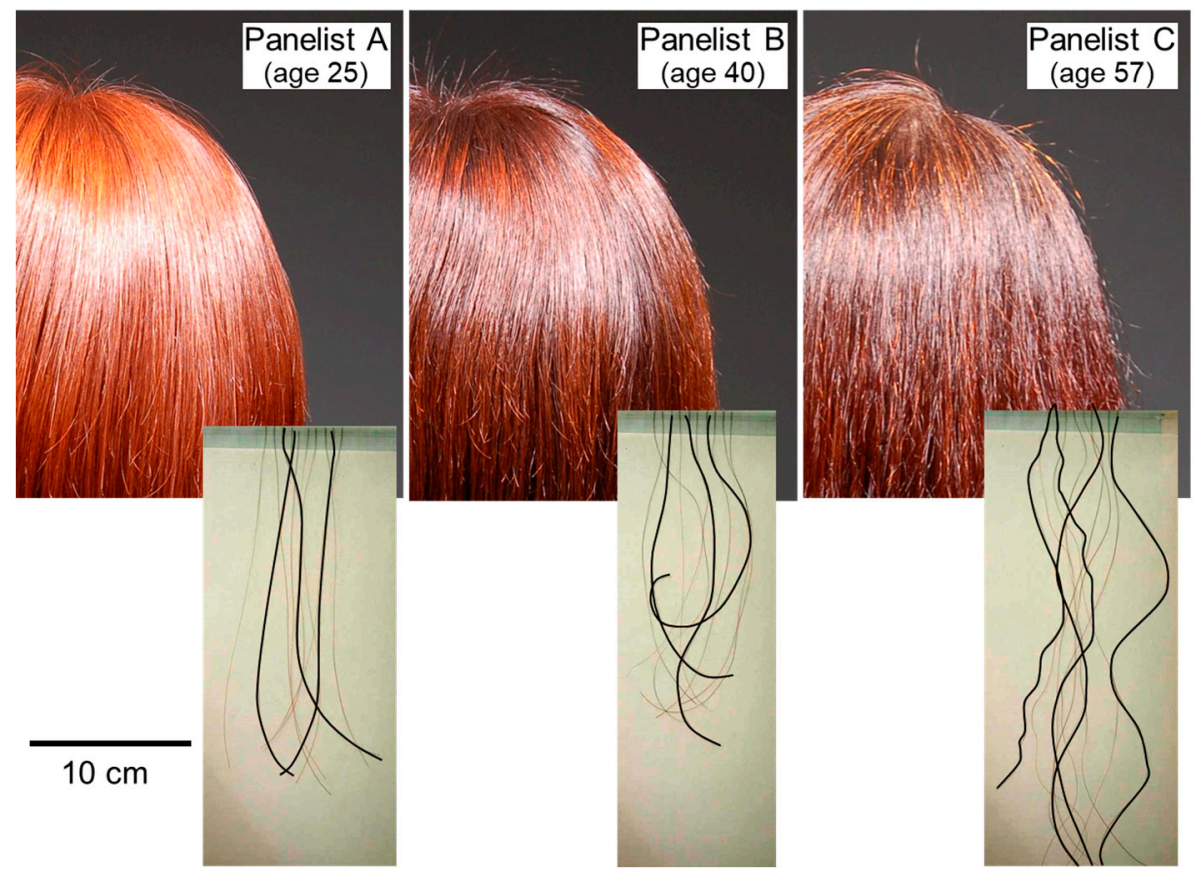

Figure 3. Hair appearances of typical panelists and the shape of corresponding hair fibers. Meandering hairs are not obvious in the panelist (A) but more in panelist (C). The alignments of multiple hair fibers are different between the panelists $(\mathbf{A}, \mathbf{C})$. The panelist $(\mathbf{B})$ shows intermediate between the panelists $(\mathbf{A}, \mathbf{C})$. This figure is quoted and revised from the references [21,22].

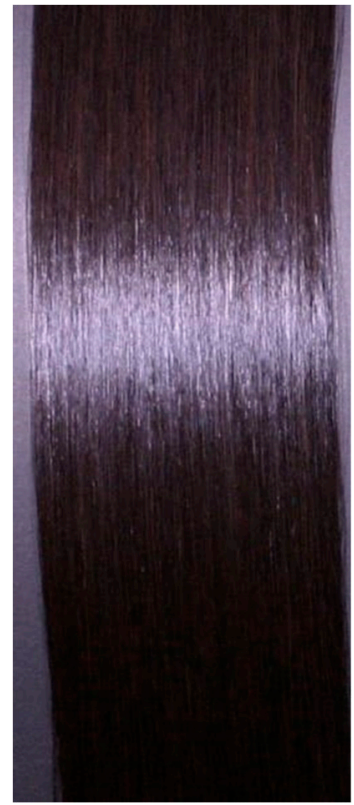

(a) Only straight hair

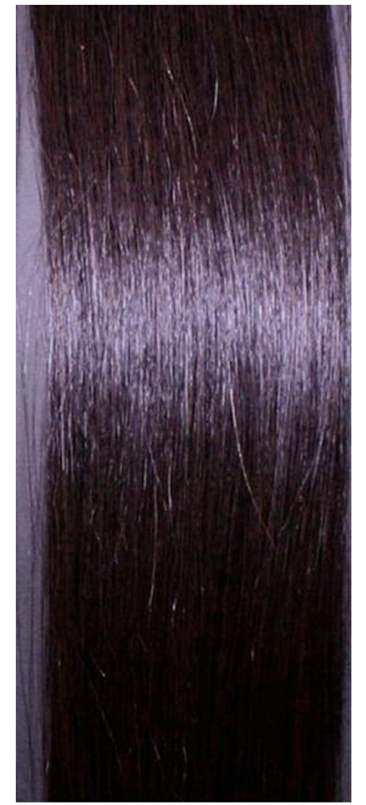

(b) 5\% Meandering hair

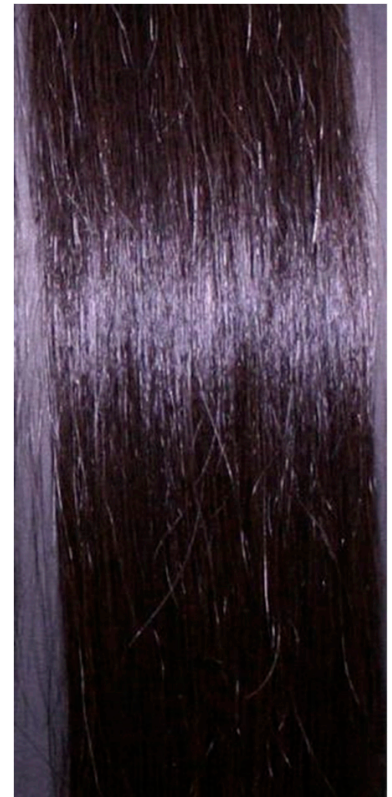

(c) 10\% meandering hair

Figure 4. Appearances of hair bundles made from straight and meandering hair fibers. Hair bundle (a) was made from only straight fibers, (b) was from the mixture of $95 \%$ straight and $5 \%$ meandering fibers, and (c) was from the mixture of $90 \%$ straight and $10 \%$ meandering fibers. Change in fiber alignment is clearly observed from more- to less-synchronized along hair length, from (a) to (c).

The alignment of multiple hair fibers highly depends on the form of each hair fiber and on the synchronicity of the neighboring hair fibers. Therefore, it is important to control hair form to modify hair appearance. The microstructure of hair fiber related to hair form has been investigated 
and a technology to control hair form has been developed. The detail of the microstructure and the technology is described in the references [25-29].

\section{Cross-Sectional Shape of Hair Fiber}

The cross-sectional shape of hair fiber is also important for hair appearance. The effect of the cross-sectional shape is summarized in article [13]. In this article, light pass in elliptical hair cross-section is discussed as shown in Figure 5. Incident light is reflected and refracted at the surface of hair fiber according to the difference in the refractive indices between hair fiber and environmental medium, usually air, based on Snell's law. The refracted incident light is reflected and refracted at the inner surface of the fiber again, and these phenomena are repeated in the fiber until the light is attenuated to a negligible level.

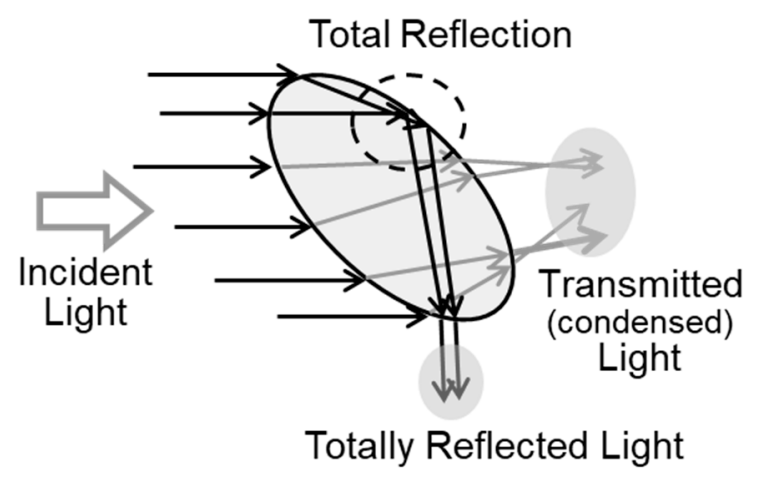

(a) Geometry of light loci in an elliptical cylinder

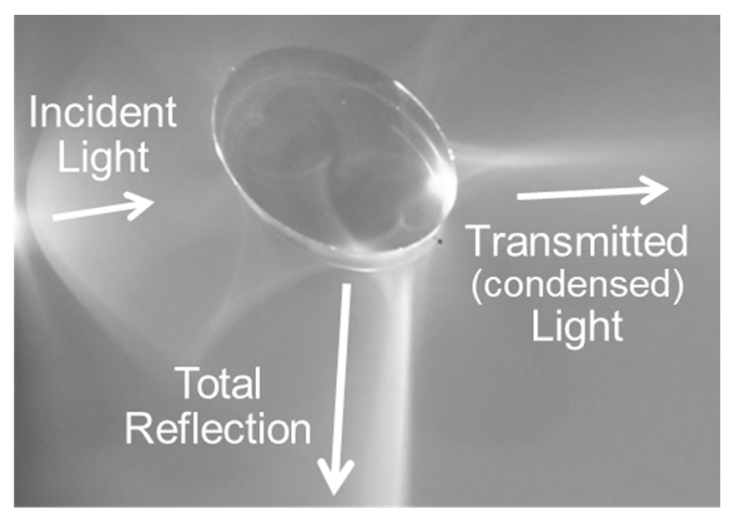

(b) Real elliptical cylinder model

Figure 5. Light reflection and refraction behaviors. (a) Shows the geometry of light loci in an elliptical cylinder, calculated with assumptions, in which ellipticity (minor/major axes) is 0.5 and the refractive index of the cylinder is 1.50. (b) Shows the light reflection and refraction behaviors of real elliptical cylinder model made of acryl resin (ellipticity is 0.7 , refractive index is ca. 1.51). This figure is quoted and revised from the references $[13,15]$.

When the incident light is refracted at an appropriate position and enters the fiber, total reflection can be observed at the inner surface, as shown in Figure 5. This total reflection is observable in the hair fiber with elliptic cross-section. The effects of the ellipticity and refractive index on the total reflection are summarized in reference [13]. The proportion of the total reflection is more in the cases of more elliptic (like western people) and in higher refractive index, and it is hard to observe the total reflection in the case of round cross-sectional shape (like eastern people).

The intensity of the total reflection is strong compared with normal reflection, and, therefore, the total reflection can be observed as glittering light from hair, especially in the case of light-colored hair such as blond hair. Several examples of the total reflection observed in real blond hair fibers are shown in reference [13].

The total reflection light passes relatively outer region of hair fiber as shown in Figure 5, and, therefore, the apparent color of the light is affected by the color of the outer region. Based on this phenomenon, an interesting technology has been proposed [30]. When the outer and inner regions in hair fiber are stained with different colors, such as blue and red as shown in the model in Figure 6a, the total reflection light passes almost only the outer region, and the color of the totally reflected light is observed as blueish and normally transmitted light reflects both inner reddish and outer blueish colors.

Figure $6 \mathrm{~b}$ shows the example of interesting hair appearance, when hair is stained with red and blue dyestuffs (HC Red 18 and HC Blue 18). The blue dyestuff (HC Blue 18) covers a larger area compared with the red dyestuff (HC Red 18), and, therefore, the blue dyestuff is more concentrated in the outer layer of hair fibers, like the model in Figure 6a. The apparent color of this hair bundle 
changes depending on the location and direction of the hair fibers, as clearly observed in Figure 6b. Figure $6 \mathrm{c}$ shows an example of a real hair cross-section stained with two different colors.

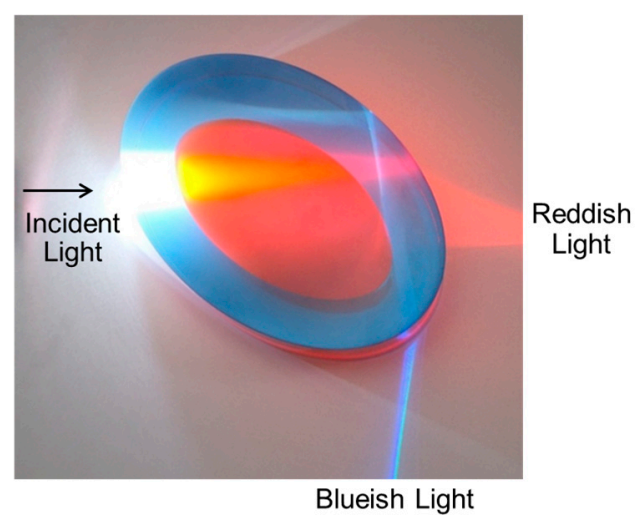

(a) Elliptic cylinder model stained two layers

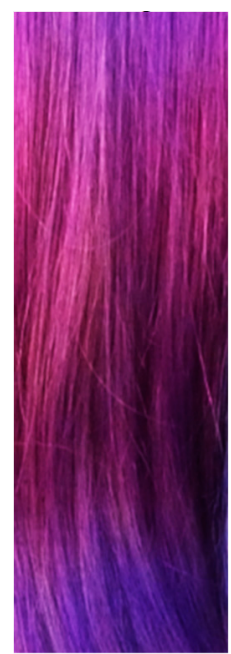

(b) Hair appearance

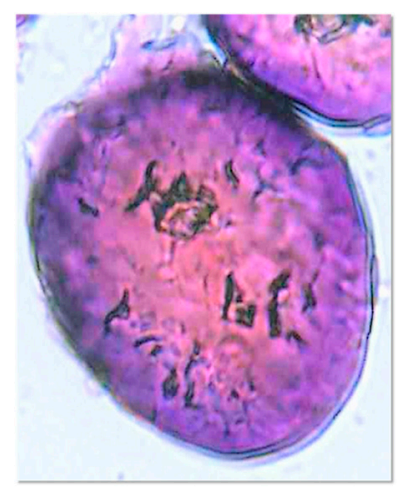

(c) Cross-section

Figure 6. Effect of light reflection and refraction on the appearance of cylindrical fibers when the cylindrical fibers are elliptical and stained two layers. (a) shows the light reflection and refraction behaviors of real elliptical cylinder model made of acryl and epoxy resins (ellipticity is 0.7 , refractive indices are ca. 1.51 and 1.57 for the acryl and epoxy resins, respectively). The outer (acryl) and inner (epoxy) layers are stained with blue and red dyestuffs, respectively. When light locus passes in the outer layer, the light reflected in the inner surface of the cylinder shows blueish color. While the light passed in the inner layer shows a more reddish color. (b) shows corresponding hair appearance. The ellipticity of hair fibers is in the range from 0.5 to 0.8 in this case. (c) is the photograph of the cross-section of real hair fiber stained with two different colors.

The above results show the importance of the cross-sectional shape of hair fiber on hair appearance in the case of light-colored hair. It is difficult to change the cross-sectional shape, but it is possible to control the penetration behavior of dyestuffs into hair fiber and to enhance the vivid appearance of hair color based on the multi-layered staining.

\section{Surface Structures of Hair Fiber}

Hair appearance is affected by the surface structure of hair fiber, which is the cuticle. In this section, the effects of cuticle structures on hair appearance are summarized based on electron and light microscopical observations. The electron microscopy reveals a change in microstructure, while the light microscopy detects corresponding light scattering parts. These microscopical methods are complementary. In addition, the effect of the inclined structure of the cuticle is discussed in relation to the unique appearance of natural human hair.

\subsection{Effect of Surface Roughness (Cuticle)}

The effect of the cuticle structure on hair appearance is summarized in Figures 7 and 8 [15,21]. The scanning electron micrographs and the light micrographs of hair fiber with relatively flat and uplifted cuticles are shown in Figure 7 and the appearances of the corresponding hair bundles are in Figure 8.

Figure 7a shows a relatively flat cuticle structure. In this case, light scattering is rarely observed as shown in Figure $7 \mathrm{~b}$ and the corresponding hair appearance shows relatively sharp and clear luster as in the left-hand side of Figure 8. On the other hand, Figure 7c shows a partially uplifted cuticle structure, because hair is excessively dried with a hot dryer after repeated shampooing more than 
100 times. In this case, light scattering is obvious as shown in Figure $7 \mathrm{~d}$ and the corresponding hair appearance shows relatively more diffused and unclear luster as in the right-hand side of Figure 8.

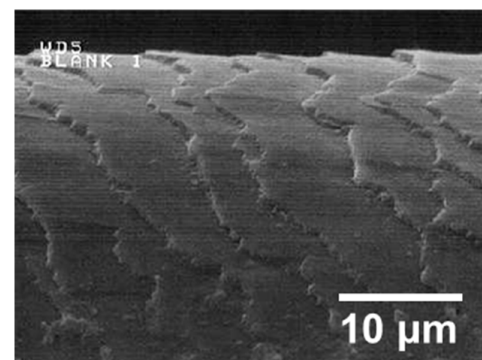

(a) SEM image of relatively flat cuticles

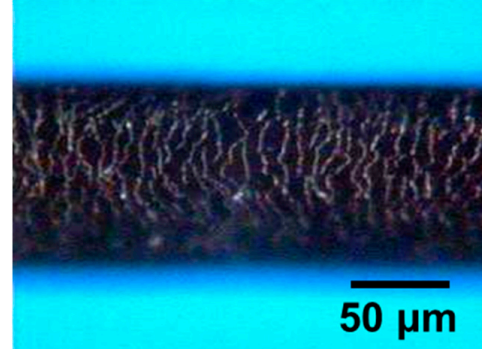

(b) Light micrograph of hair fiber with relatively flat cuticles

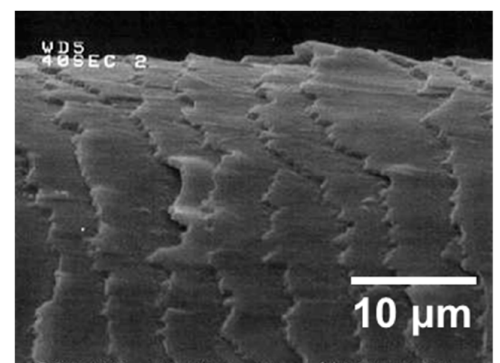

(c) SEM image of uplifted cuticles

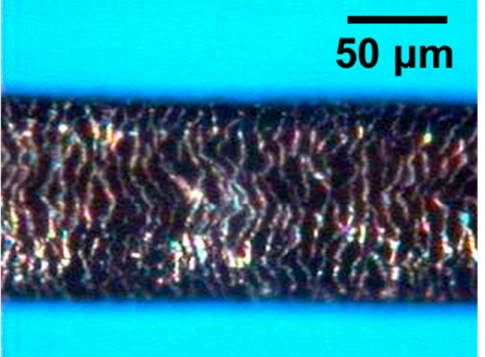

(d) Light micrograph of hair fiber with uplifted cuticles

Figure 7. Micrographs of hair fibers with relatively flat and uplifted cuticles, those were taken by a scanning electron microscopy (SEM) and light microscopy. (a,b) are SEM image and light micrograph of hair fiber with relatively flat cuticle, and (c,d) are those of hair fiber with uplifted cuticles. In the light microscopy, any mounting medium was not used to observe light scattering under ambient conditions. Those figures are quoted and revised from the references $[15,21]$.

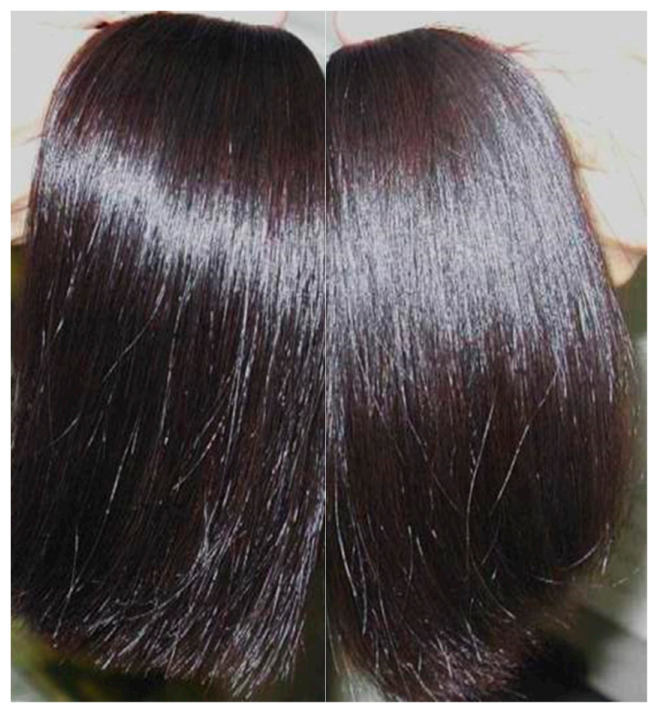

Left: flat cuticle/Right: uplifted cuticle

Figure 8. The appearances of hair bundles with relatively flat and uplifted cuticles. Left and right-hand sides are hair bundles with flat and uplifted cuticles, respectively. In order to compare the bundles under the same lighting conditions as possible, the light source was located over the hair bundles at equal distance from each bundle, and hair form was kept as similar as possible. This figure is quoted and revised from the references $[15,21]$.

These results clearly show that the surface structure of the cuticle is important for hair appearance via light scattering behavior at the surface cuticle. In order to control the surface conditions of hair 
fibers, various technologies have been developed so far. One of the technologies is covering the hair surface with chemical materials such as oils and polymers [7,8]. These technologies are effective to smoothen the hair surface and enhance hair luster; however, sometimes undesirable change in the hair appearance is caused by over-covering, such as excessive glare and artificial impression. One of the reasons for the artificial impression is discussed in the following sub-section, regarding the effect of an inclined cuticle structure.

Other technologies to control surface conditions have been proposed [11,31]. A technology using malic acid is described in reference [11]. When damaged hair with a rough surface is treated with the malic acid, the structures of hair are swollen by the penetration of malic acid and a smooth surface is recovered through the swelling. No obvious materials remain on the hair surface, and, therefore, over-conditioning does not occur in this case. In reference [31], the formation of a fine structure on the hair surface is described. When the size of the fine structure is less than the wavelength of visible light, the reflection at the surface is suppressed and the apparent hair color is enhanced, similar to the moth-eye effect [32].

\subsection{Effect of an Inclined Cuticle Structure}

The cuticle of hair has another effect on hair appearance based on its inclined structure $[10,15]$. The cuticle layers are scaly and inclined one direction from root to tip ends, like in Figure 9b. The inclined angle is around 2.5 degree [6]. Therefore, specular reflections at the front and back surfaces are observed at different positions. The front and back surface reflections move downward and upward, respectively, in the case of the optical system shown in Figure 9b. The color of the front surface reflection is almost the same as the incident light, which is usually white, because there is no colorant in the light locus from the incident light to the eye. On the other hand, the color of the back surface reflection well-reflects the color of internal hair fiber because the light penetrates into hair fiber as shown in Figure $9 \mathrm{~b}$. Based on the above phenomena, we can perceive the double reflections (colored and not-colored), as in Figure 9a.

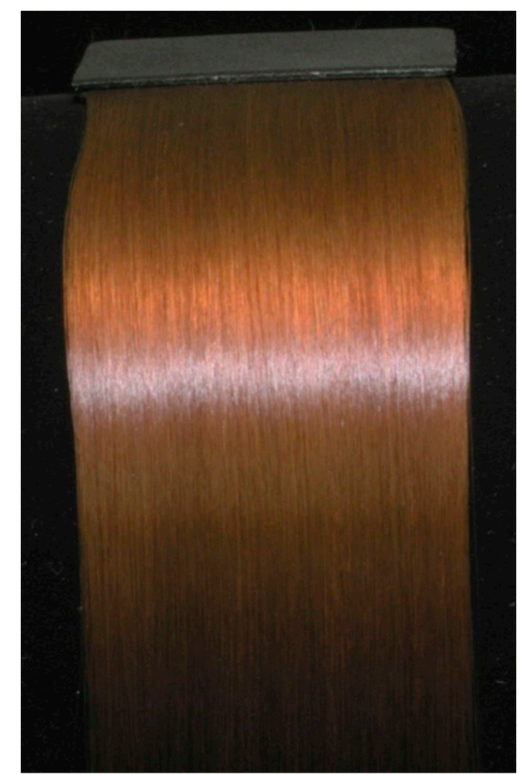

(a) Fibers with an inclined cuticle structure

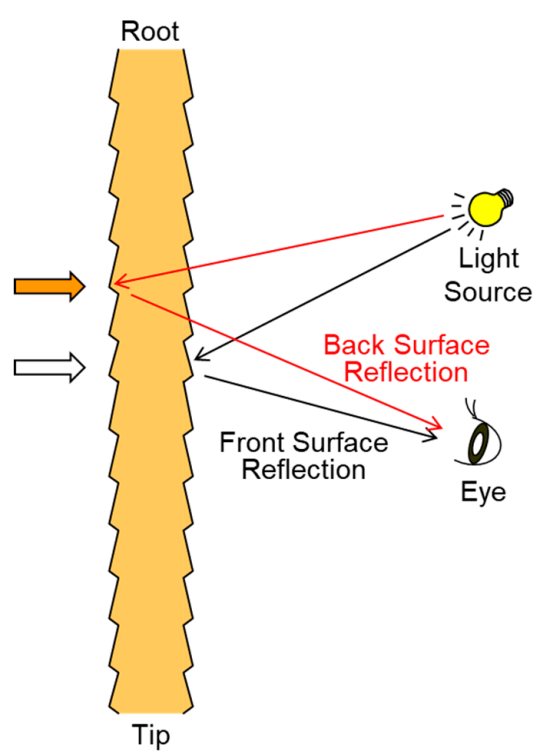

(b) Front and back surface reflections

Figure 9. The effect of an inclined cuticle structure on hair appearance. (a) is the appearance of real human hair, which has inclined cuticle structure. The surface of the human hair fiber is tilted around 2.5 degrees against to fiber axis because of layered cuticle structure. (b) shows a schematic model to understand light loci from a light source to an eye via single hair fiber, corresponding to the hair with an inclined cuticle structure. 
Those double reflections are not observable in the case of the cuticle-less hair, such as highly damaged de-cuticled hair and artificial hairs. Figure 10 shows the appearance of the artificial hair made of nylon fiber and the schematic model of the optical system in this case. The specular reflections at the front and back surfaces of the fiber are observed at almost the same position as shown in Figure 10b, especially when the fiber thickness is very thin compared with the distance between the hair fiber and the eye. The thickness of hair fiber is usually less than $150 \mu \mathrm{m}$ and the fiber-eye distance is more than $150 \mathrm{~mm}$ in most case; therefore, the front and back surface reflections look like a single reflection as shown in Figure 10a. The apparent single reflection is slightly colored because this reflection is a mixture of the non-colored surface reflection and the colored back surface reflection. Such a colored reflection gives a metallic impression. This is probably one reason behind the artificial impression of artificial hair. The similar phenomenon is observed in the case of over-covering with oils and/or polymers on the hair surface, as described in the previous sub-section.

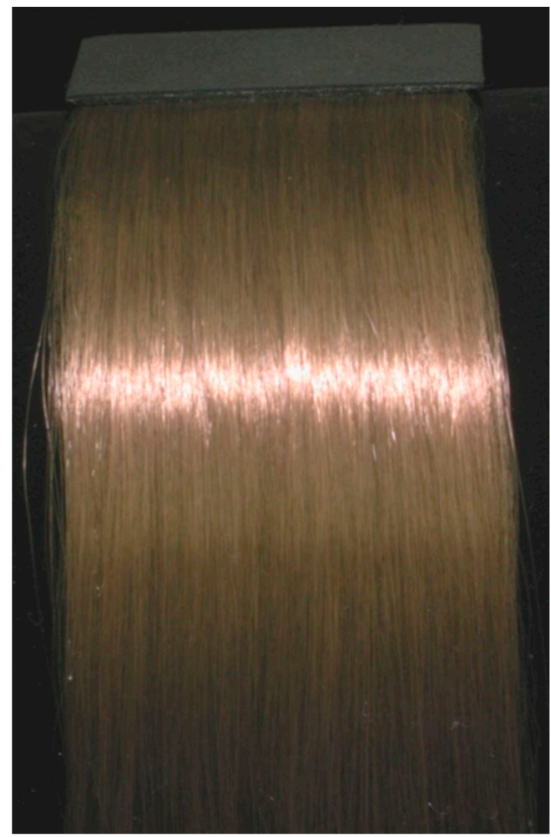

(a) Fibers without inclined cuticle

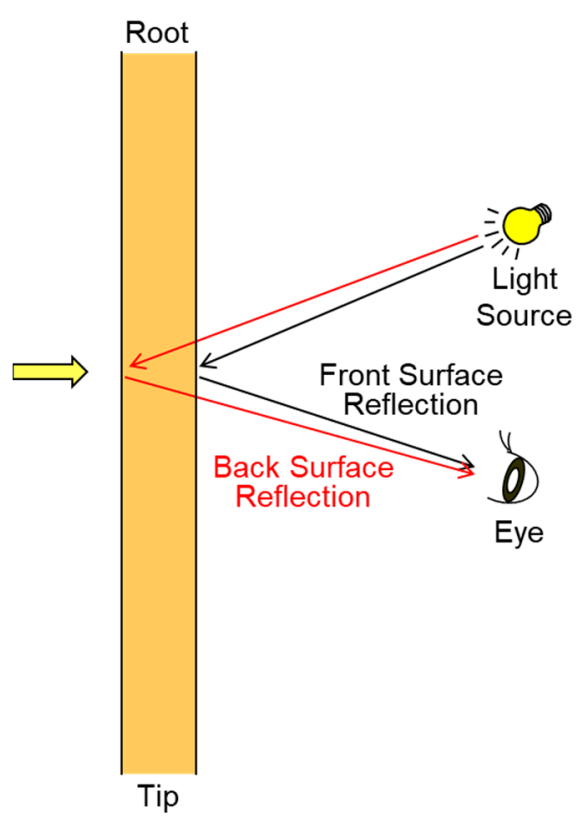

(b) Front and back surface reflections

Figure 10. The effect of the not-inclined surface of fiber on fiber appearance. (a) is the appearance of artificial nylon hair fiber, which has no inclined cuticle structure. The surface of the artificial fiber is basically flat and parallel to the fiber axis. (b) shows a schematic model to understand light loci from a light source to an eye via single fiber, corresponding to the fiber without cuticle.

The above phenomena show the importance of an inclined cuticle structure on the natural appearance of hair. It is difficult to control the inclined structure, especially for de-cuticled hair, and, therefore, it is important to keep the inclined cuticle structure in its original state to retain a natural look.

\section{Internal Structures of Hair Fiber}

When hair color is not so dark, hair appearance can be affected by internal hair structures, such as the cortex and medulla $[9,10,12-16,21]$. In this section, the effects of those structures on hair appearance are summarized based on electron and light microscopical observations. Electron microscopy reveals changes in the microstructure, while light microscopy detects corresponding light scattering parts, as well as the previous section for the surface structure. 


\subsection{Appearance of Transparent (Pore-less) Hair Fiber}

When there are small differences in refractive indices in hair fiber, light scattering in the fiber could be low level, that is, the hair fiber can be almost transparent. Figure 11a shows the typical example of the appearance of such almost transparent hair fibers. Figure $11 \mathrm{~b}, \mathrm{c}$ are the scanning electron micrograph and the light micrograph of corresponding almost transparent hair fiber $[9,10,15,21]$.

There is no porous structure in the fiber (medulla and cortex regions) in this case as in Figure 11b, and light scattering is kept at a low level in Figure 11c, other than slight light scattering at the cuticle edges. The colored brighter part in Figure 11c is a specular reflection at the back surface of hair fiber as described in Figure 9b. The diffuse reflection is, therefore, kept at a lower level in this case.

As a result of the lower diffuse reflection, the hair looks lustrous with higher contrast. The double reflections of colored and non-colored highlight are clearly observed as shown in Figure 11a. The apparent hair color looks darker but vivid. This appearance of pore-less hair is compared with that of porous hair in the following sub-sections.

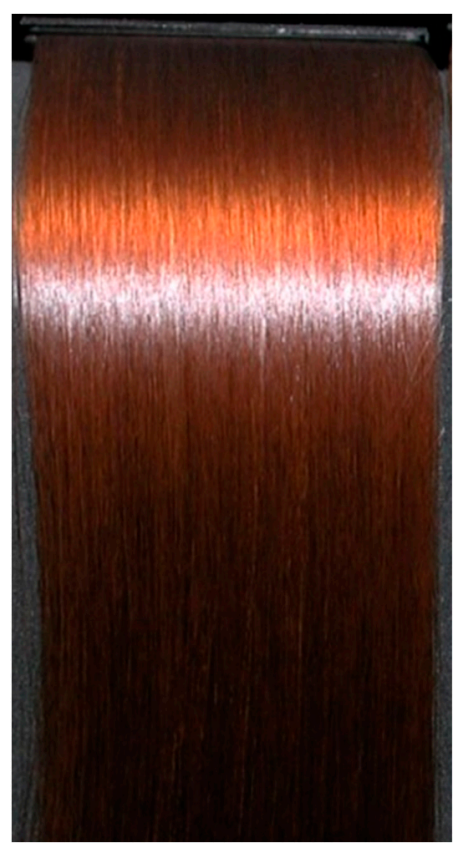

(a) Hair appearance

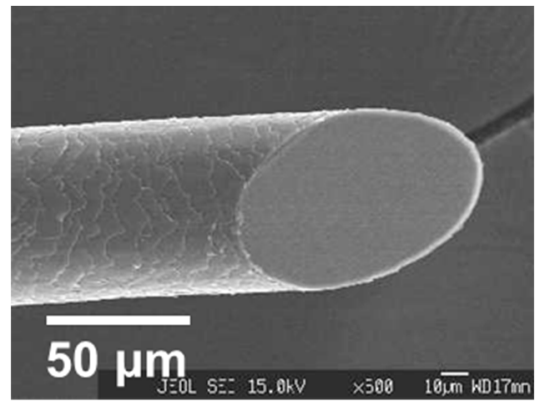

(b) SEM image

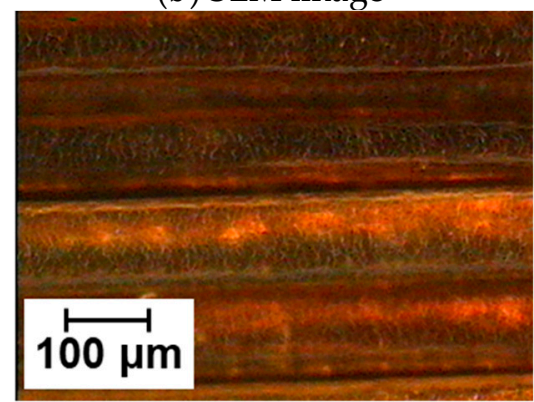

(c) Light micrograph

Figure 11. Hair appearance and the corresponding micrographs of the hair with a pore-less structure in the cortex and medulla. (a) is hair appearance, $(\mathbf{b}, \mathbf{c})$ is scanning electron micrograph (SEM) and light micrograph for corresponding hair fibers. In light microscopy, any mounting medium was not used to observe light scattering under ambient conditions. These pictures are quoted and revised from the references [15,21].

\subsection{Effect of Porous Medulla}

The effect of porous medulla on hair appearance is shown in Figure 12a, and the corresponding micrographs of scanning electron and light microscopies are in Figure $12 b, c$, respectively $[9,10,15,21]$. The porous structure of the medulla is clearly observed at the center of hair fiber as in Figure 12b. In Figure 12c, brighter and whitish lines are observable at the center of hair fibers. Those are light scattering at the porous medulla. There is no obvious light scattering other than at the medulla, so the cortex is still kept as relatively transparent in this case.

As a result of the light scattering at the porous medulla, the corresponding hair appearance in Figure 12a shows brighter color with lower contrast compared with the appearance of almost transparent hair in Figure 11a. The difference in the hair appearances between Figures 11 and 12 is 
caused only by the amount of the porous structure of the medulla. These results indicate the importance of the light scattering at the porous structure of the medulla on hair appearance.

Interestingly, the light scattering at the porous structure of the medulla is reversibly changed from more- to less-scattered states by the swelling of the hair fiber from dry to wet states in water, and from less- to more-scattered states by drying from wet to dry states again. Those reversible changes from less- to more-scattered states are shown in Figure 13 according to the drying time from 0 to 8 min with a hot dryer. The bright lines of light scattering at the porous medulla in hair fibers are rarely observed or slightly observed at 0 min (under wet condition), but the bright lines gradually increase with drying time, as shown in the figures.

These reversible changes in the light scattering at the porous structure by wetting and drying processes suggest that the light scattering can be controlled by the swelling of the hair structure. Based on the above results, a lot of chemicals were investigated in terms of the swelling of hair structure to control the light scattering at the medulla. As a result of this investigation, malic and succinic acids were found as the candidates of effective chemicals $[10,11]$.

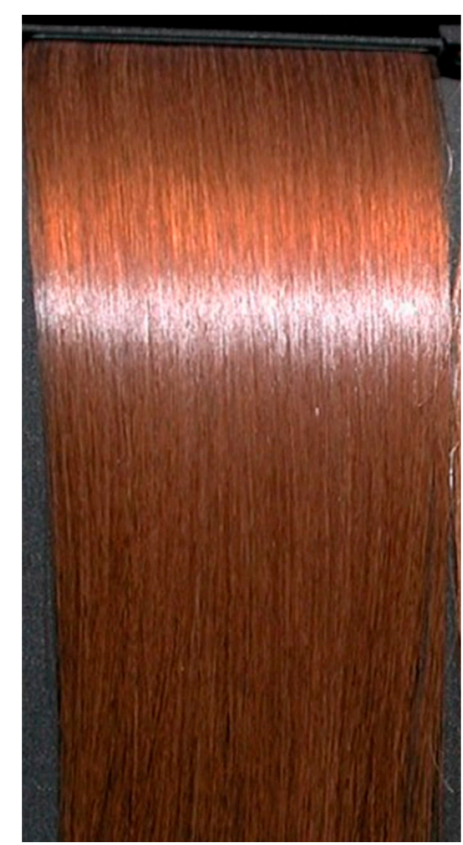

(a) Hair appearance

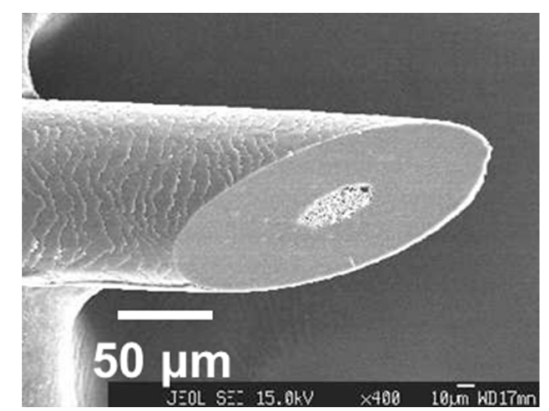

(b) SEM image

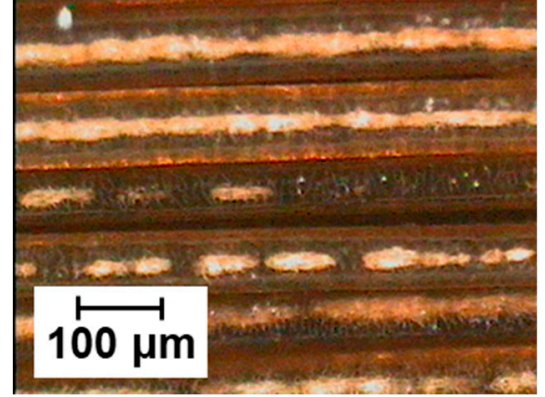

(c) Light micrograph

Figure 12. Hair appearance and the corresponding micrographs of the hair, which shows porous structure in the medulla. (a) is hair appearance, $(\mathbf{b}, \mathbf{c})$ is scanning electron micrograph (SEM) and light micrograph for corresponding hair fibers. In the light microscopy, any mounting medium was not used to observe light scattering under ambient conditions. These pictures are quoted and revised from the references $[15,21]$.

\subsection{Effect of Micro-Pores in the Cortex}

The typical effect of the cortex structure on hair appearance is shown in Figure 14. When hair is highly damaged by excessive coloring and repeated shampooing, a lot of micro-pores are generated in the cortex as shown in Figure $14 \mathrm{~b}$ and hair fibers become turbid as in Figure 14c because of light scattering at the micropores. Two types of light scatterings are observed in this figure. One is at the cortex and the other is at the medulla. As a result of those light scatterings, further changes in the apparent color and contrast are observed in the hair appearance in Figure 14a compared with Figure 12a. The apparent color and contrast become lighter and lower, respectively, according to the degree of light scattering in the order of "pore-less (Figure 11) < porous medulla (Figure 12) < micro-pores in the cortex, as seen in Figure 14" [10,15,21]. 


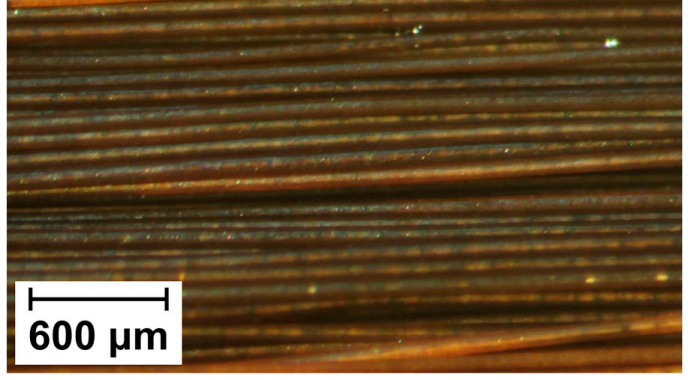

(a) Drying time 0 min (wet state)

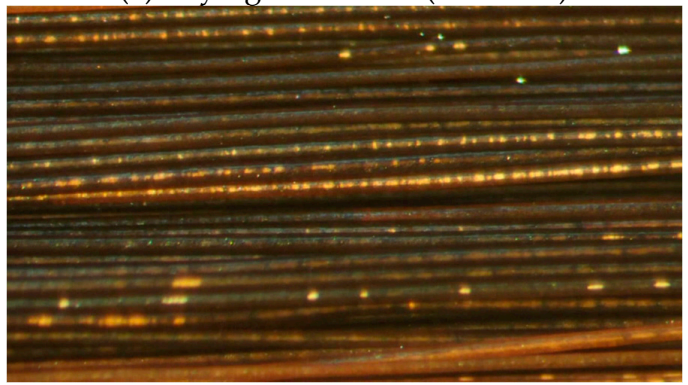

(b) Drying time $1 \mathrm{~min}$

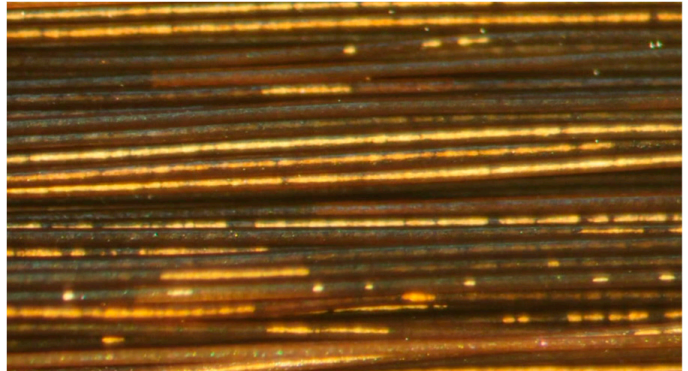

(c) Drying time 4 min

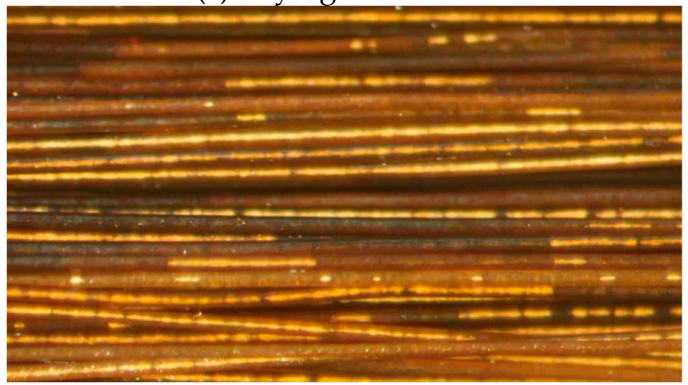

(d) Drying time 8 min

Figure 13. Changes in light scattering at the porous structure of the medulla from wet to dry states. (a-d) show the changes in the light scattering from 0 to $8 \mathrm{~min}$ of drying time with a hot dryer. In the light microscopy, any mounting medium was not used to observe only the effects of water and air on the light scattering under ambient conditions.

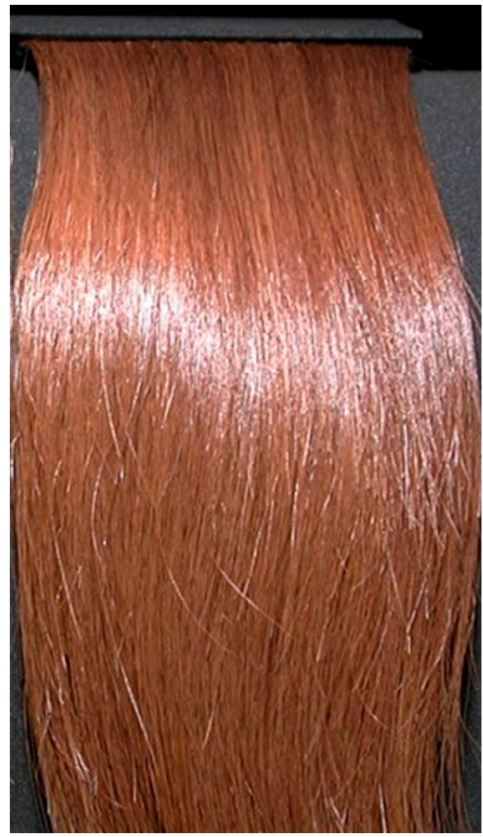

(a) Hair appearance

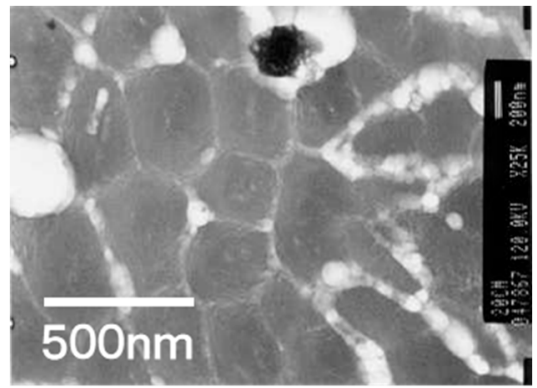

(b) TEM image

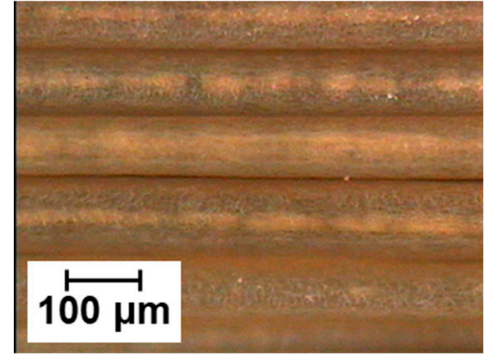

(c) Light micrograph

Figure 14. Hair appearance and the corresponding micrographs of the hair, which shows micro-pores in the cortex. (a) is hair appearance, $(\mathbf{b}, \mathbf{c})$ is transmission electron micrograph (TEM) and light micrograph for corresponding hair fibers. In light microscopy, any mounting medium was not used to observe light scattering under ambient conditions. These pictures are quoted and revised from the references [15,21].

The light scattering at the micro-pores in the cortex can be controlled by the swelling of hair structures by water, as well as the light scattering at the porous structure of the medulla. Organic acids 
like malic and succinic acids are also effective to control the light scattering at the micro-pores in the cortex also [10].

\section{Conclusions}

The above results of the effects of various hair structures on hair appearance are summarized in this section and possible conditions to control hair appearance are considered as a conclusion.

\subsection{Effects of Various Hair Structures on Hair Appearance}

The typical effects of various hair structures are shown in the previous sections from the macroscopic form and alignment of multiple hair fibers to microscopic porous structures.

The form of hair fiber is effective for hair appearance via the alignment of the multiple fibers. A hair bundle with more-synchronized hair fibers shows sharp and clear hair luster, while a bundle with less-synchronized hair fibers shows broad and unclear luster as shown in Figures 3 and 4 . The synchronicity of multiple fibers can be considered as an important factor to control hair appearance. The importance of the fiber synchronicity is not only for straight hair style, but also for curved hair style, like wavy and curly. In the cases of wavy and curly hair, the fiber synchronicity is described as "frizzy" or "defined" by consumers. The "frizzy" and "defined" correspond to less-synchronized and well-synchronized, respectively, and are important factors for the consumers.

The cross-sectional shape of hair fiber affects the shining and/or glittering behavior of hair fiber. The elliptic cross-section is effective for that behavior via the total reflection at the inner surface of the hair fiber, especially in the case of light-colored hair such as blond hair, as described in Figure 5. In addition, the combination of the elliptic cross-section and multi-layered staining in hair fiber results in an interesting multi-tonal appearance as in Figure 6. The cross-sectional shape and multi-layered staining are probably important for the enhancement of shining and colorful impressions, respectively.

The cuticle structure of hair fiber has significant effects on hair appearance. The surface roughness of the cuticle affects hair luster caused by the specular reflection at the surface, as shown in Figures 7 and 8 . The inclined structure of the cuticle is effective for the natural or artificial impressions via the double reflections from the front and back surfaces of the fiber, as in Figures 9 and 10. Those results mean that an ordered cuticle structure is important for hair luster and natural impression.

The internal microstructures of hair fiber are important for apparent hair color and luster. Porous structure generated in the medulla and/or cortex causes light scattering in hair fiber, and, therefore, the apparent hair color and luster become lighter and broader, respectively, as shown in Figures 11, 12 and 14. The pore-less (less-scattering) structure of hair fiber is important for clear hair color and sharp luster with higher contrast.

\subsection{Conditions to Control Hair Appearance}

Based on the above summary, I consider the following conditions to control hair appearance:

1. Synchronized alignment of multiple hair fibers,

2. Appropriate hair cross-sectional shape and distribution of colorants in hair fiber,

3. Ordered inclined cuticle layers,

4. Pore-less (less-scattering) internal structures in the cortex and medulla

The above conditions should be modified and revised according to new findings in the future. However, I think that those conditions are useful hints for the development of effective technologies to control hair appearance.

Funding: This research received no external funding.

Acknowledgments: I would like to thank all collaborators internal and external Kao Corporation for their significant contributions.

Conflicts of Interest: The authors declare no conflict of interest. 


\section{References}

1. Robbins, C.R. Chemical and Physical Behavior of Human Hair, 5th ed.; Springer: Berlin, Germany, 2012; pp. 677-685.

2. Stamm, R.F.; Garcia, M.L.; Fuchs, J.J. The optical properties of human hair. I. Fundamental consideration and goniophotometer curves. J. Soc. Cosmet. Chem. 1977, 28, 571-599.

3. Stamm, R.F.; Garcia, M.L.; Fuchs, J.J. The optical properties of human hair. II. The luster of hair fiber. J. Soc. Cosmet. Chem. 1977, 28, 601-609.

4. Maeda, T.; Okada, M.; Watanabe, H.; Hara, T. Measurements of hair luster by color image analysis. J. Soc. Cosmet. Chem. Jpn. 1992, 25, 215-222. [CrossRef]

5. Guiolet, A.; Garson, J.C.; Levecque, J.L. Study of the optical properties of human hair. Int. J. Cosmet. Sci. 1987, 9, 111-124. [CrossRef] [PubMed]

6. Wortmann, F.-J.; Schulze zur Wiesche, E.; Bierbaum, A. Analyzing the laser-light reflection from human hair fibers. I. Light components underlying the goniophotometric curves and fiber cuticle angles. J. Cosmet. Sci. 2003, 54, 301-316.

7. McMullen, R.; Jachowicz, J. Optical properties of hair: Effect of treatments on luster as quantified by image analysis. J. Cosmet. Sci. 2003, 54, 335-351. [PubMed]

8. Puccettii, G.; Thompson, W. Effects of hair sprays on colour perception: A hyperspectral imaging approach to shine and chroma on heads. Int. J. Cosmet. Sci. 2017, 39, 156-164. [CrossRef] [PubMed]

9. Nagase, S.; Shibuichi, S.; Ando, K.; Kariya, E.; Satoh, N. Influence of internal structure of hair fiber on hair appearance. I. Light scattering from the porous structure of the medulla of human hair. J. Cosmet. Sci. 2002, $53,89-100$.

10. Nagase, S.; Satoh, N.; Nakamura, K. Influence of internal structure of hair fiber on hair appearance. II. Consideration of the visual perception mechanism of hair appearance. J. Cosmet. Sci. 2002, 53, 387-402.

11. Okamoto, M.; Yakawa, R.; Mamada, A.; Inoue, S.; Nagase, S.; Shibuichi, S.; Kariya, E.; Satoh, N. Influence of internal structure of hair fiber on hair appearance. III. Generation of light-scattering factors in hair cuticles and the influence on hair shine. J. Cosmet. Sci. 2003, 54, 353-366.

12. Keis, K.; Ramaprasad, R.; Kamath, Y.K. Effect of hair color on luster. J. Cosmet. Sci. 2004, 55, 423-436. [CrossRef] [PubMed]

13. Iwai, S.; Sato, H.; Shibuichi, S.; Satoh, N. Novel mechanism for hair shine with special optical enhancement effect on blond hair. J. Cosmet. Sci. 2009, 60, 323-336. [PubMed]

14. Kharin, A.; Varghese, B.; Verhagen, R.; Uzunbajakava, N. Optical properties of the medulla and the cortex of human scalp hair. J. Biomed. Opt. 2009, 14, 024035. [CrossRef] [PubMed]

15. Nagase, S.; Shibuichi, S. Beautiful hair appearance and hair structure. Jpn. J. Opt. 2010, 39, 518-523.

16. Yamazaki, J.; Maeda, K. Analysis of lipids in the medulla of Japanese hair and their function. Cosmetics 2018, 5, 27. [CrossRef]

17. Wortmann, F.-J.; Schulze zur Wiesche, E.; Bourceau, B. Analyzing the laser-light reflection from human hair fibers. II. Deriving a measure of hair luster. J. Cosmet. Sci. 2004, 55, 81-93. [CrossRef]

18. Lefaudeux, N.; Lechocinski, N.; Clemenceau, P.; Breugnot, S. New luster formula for the characterization of hair tresses using polarization imaging. J. Cosmet. Sci. 2009, 60, 153-169. [CrossRef]

19. Marschner, S.R.; Jensen, H.W.; Cammarano, M.; Worley, S.; Hanrahan, P. Light scattering from human hair fibers. ACM Trans. Graph. 2003, 22, 780-791. [CrossRef]

20. McMullen, R.; Jachowicz, J. Optical properties of hair-Detailed examination of specular reflection patterns in various hair types. J. Cosmet. Sci. 2004, 55, 29-47. [CrossRef]

21. Nagase, S. Hair structure and properties: Introduction to recent works. J. Jpn. Cosmet. Sci. Soc. 2008, 32, 214-220.

22. Nagase, S.; Kajiura, Y.; Mamada, A.; Abe, H.; Shibuichi, S.; Satoh, N.; Itou, T.; Shinohara, Y.; Amemiya, Y. Change in structure and geometric properties of human hair by aging. J. Cosmet. Sci. 2009, 60, 637-648. [CrossRef] [PubMed]

23. Fink, B.; Liebner, K.; Müller, A.-K.; Hirn, T.; McKelvey, G.; Lankhof, J. Hair colour and skin colour together influence perceptions of age, health and attractiveness in lightly pigmented young. Int. J. Cosmet. Sci. 2018, 40, 303-312. [CrossRef] [PubMed] 
24. Tanamachi, H. Temperature as a moisture cue in haptics on hair. Int. J. Cosmet. Sci. 2011, 33, 25-36. [CrossRef] [PubMed]

25. Kajiura, Y.; Watanabe, S.; Itou, T.; Nakamura, K.; Iida, A.; Inoue, K.; Yagi, N.; Shinohara, Y.; Amemiya, Y. Structural analysis of human hair single fibres by scanning microbeam SAXS. J. Struct. Biol. 2006, 155, 438-444. [CrossRef] [PubMed]

26. Nagase, S.; Tsuchiya, M.; Matsui, T.; Shibuichi, S.; Tsujimura, H.; Masukawa, Y.; Satoh, N.; Itou, T.; Koike, K.; Tsujii, K. Characterization of curved human hair of Japanese women with reference to internal structures and amino acid composition. J. Cosmet. Sci. 2008, 59, 317-332. [PubMed]

27. Bryson, W.G.; Harland, D.P.; Caldwell, J.P.; Vernon, J.A.; Walls, R.J.; Woods, J.L.; Nagase, S.; Itou, T.; Koike, K. Cortical cell types and intermediate filament arrangements correlate with fiber curvature in Japanese human hair. J. Struct. Biol. 2009, 166, 46-58. [CrossRef] [PubMed]

28. Harland, D.P.; Vernon, J.A.; Woods, J.L.; Nagase, S.; Itou, T.; Koike, K.; Scobie, D.A.; Grosvenor, A.J.; Dyer, J.M.; Clerens, S. Intrinsic curvature in wool fibres is determined by the relative length of orthocortical and paracortical cells. J. Exp. Biol. 2018, 221, 172312. [CrossRef] [PubMed]

29. Bryson, W.G.; Harland, D.P.; Caldwell, J.P.; Vernon, J.A.; Walls, R.J.; Woods, J.L.; Nagase, S.; Itou, T.; Koike, K. Electron microscopy and tomography reveal that sodium 2-naphthalene sulfonate incorporated into perming solutions swells and tilts trichocyte intermediate filaments causing straightening of curly Japanese human hair. Int. J. Cosmet. Sci. 2019, 41, 132-146. [CrossRef]

30. Nakamura, T.; Nagase, S.; Nojiri, M.; Breakspear, S.; Ishiwata, Y.; Fujita, A.; Hartmann, H. High definition dissociative delocalized dyes: A new class of physical hair dyes with unique 3D reflection on hair. In Proceedings of the 30th IFSCC Congress, Munich, Germany, 18-21 September 2018. O-S7-05.

31. Watanabe, S.; Sato, H.; Shibuichi, S.; Okamoto, M.; Inoue, S.; Satoh, N. Formation of nanostructure on hair surface: Its characteristic optical properties and application to hair care products. J. Cosmet. Sci. 2007, 58, 283-294.

32. Han, Z.W.; Wang, Z.; Feng, X.M.; Li, B.; Mu, Z.Z.; Zhang, J.Q.; Niu, S.C.; Ren, L.Q. Antireflective surface inspired from biology: A review. Biosurf. Biotribol. 2016, 2, 137-150. [CrossRef]

(C) 2019 by the author. Licensee MDPI, Basel, Switzerland. This article is an open access article distributed under the terms and conditions of the Creative Commons Attribution (CC BY) license (http://creativecommons.org/licenses/by/4.0/). 